\title{
USE OF MITOTIC SEGREGATION FOR ASSIGNING GENES TO LINKAGE GROUPS IN ASPERGILLUS NIDULANS
}

\author{
E. FORBES \\ Department of Genetics, University of Glasgow
}

\section{INTRODUCTION}

Received 22.v. $5^{8}$

THE difficulties encountered in the early stages of mapping the chromosomes of some organisms can be reduced by using mitotic segregation and recombination. In Aspergillus nidulans mitotic segregation gives rise to two types of segregants; diploids produced as a consequence of mitotic crossing over, and haploids produced as a consequence of a process as yet not well understood and which for short has been called haploidisation (Pontecorvo, Tarr-Gloor and Forbes, I954; Pontecorvo and Käfer, 1956, 1958).

Haploid segregants arising from a multiply marked heterozygous diploid carry either one or the other member of each homologous chromosome pair of the parent diploid. As haploidisation does not involve crossing over, whole chromosomes segregate as units and recombine as units with other non-homologous chromosomes. On the other hand, diploid segregants arising from the same heterozygous diploid as a consequence of mitotic crossing over are usually homozygous for only one chromosome segment (distal to the position of crossing over) and still heterozygous for the rest of the other markers. The two types of segregants can be distinguished from one another by means of various criteria. The frequency of both types is low, the haploids usually in the minority.

A diploid heterozygous for one marker in each linkage group gives haploid segregants showing all combinations of these unlinked markers. On the other hand, two markers in the same linkage group but in the "repulsion" (" trans ") arrangement will not usually appear together among haploid segregants. This means that an unlocated mutant reveals its linkage group by not appearing together with the marker with which it is in repulsion. Thus the problem of identifying the linkage group of an unlocated mutant is that of selecting haploid segregants and classifying them for recombination or failure of recombination between the unlocated mutant and each of the tester markers.

Since haploid segregants are rare, methods facilitating selection are necessary. A possible way to do this is to take advantage of the fact that haploid segregants show free recombination between markers on different chromosome pairs and complete linkage between markers on the same chromosome pair. Thus, among haploid segregants showing a recessive or incompletely dominant marker one half show another unlinked recessive or incompletely dominant marker. In contrast to this, among diploid segregants only a fraction of one per 
cent. of those homozygous for one recessive or incompletely dominant marker are homozygous for another recessive or incompletely dominant marker on a different chromosome pair or on a different arm of the same pair.

Thus, if we select simultaneously for two recessive or semi-dominant markers either on different chromosomes or on different arms of the same chromosome, we select mainly haploids. Among them the proportion of diploid segregants homozygous for both markers will be negligible.

The markers for this "combined selection" (Käfer, 1958, p. II4) should lend themselves to automatic screening. For example, a partially dominant marker AcrI in heterozygous condition AcrI $/+$ confers. partial resistance to acriflavine. The homozygote Acri/AcrI and the haploid AcrI are resistant to a higher concentration of acriflavine and can therefore be automatically selected on medium with high acriflavine concentration (Roper and Käfer, I957).

As another example, a recessive suppressor suIadzo (which will be abbreviated to $s u$ ) in homozygous or haploid condition allows normal growth of homozygotes $a d 20 / a d 2 o$ or haploid $a d 2 o$ on adenineless medium on which the parent diploid $\frac{\operatorname{suI} a d_{20}}{+} \frac{a d_{20}}{a d 2 o}$ grows poorly (Pritchard, I955).

As a third example, segregants $w$ or $w / w$ (with colourless, "white" conidia) from a diploid $w /+$ (coloured conidia) can be identified by inspection and isolated. So also can $y$ or $y / y$ segregants (yellow conidia) from a diploid $y /+$ (green). ( $w$ is epistatic to $y$ and $y^{+}$.)

A combined selection, on adenineless medium with an adequate concentration of acriflavine, from a $\frac{s u}{+} \frac{a d 20}{a d 2 o} \frac{A c r I}{+}$ heterozygous diploid, will pick out haploid segregants su adro Acri and a few diploid segregants $\frac{\text { su ad2o }}{\text { su ad2o }} \frac{\text { AcrI }}{\text { AcrI }}$

Another combined selection, on adenineless medium, can screen su ad2o w (adenine independent, colourless) from $\frac{s u \text { ad2o }}{+\quad a d 2 o} \frac{w}{+}$. The screening is automatic in respect to $s u$, and visual in respect to $w$.

The technical requirements indicated above-automatic selection of haploids and one marker in each linkage group besides those required. for selection-have been partially fulfilled by preparing two haploid tester strains. They were obtained by combining the required markers by appropriate crosses. A haploid strain carrying an unlocated mutant can be combined with either of these tester strains to form a heterozygous diploid. The two tester strains prepared so far do not have all eight linkage groups marked, but one (Tester Strain A) has linkage groups I, II, III, IV, V and VII marked, and the other (Tester Strain B) linkage groups I, II and VI. Any mutant, which fails to be 


\section{TABLE}

Mutants used in the present work

(data from various sources)

Chromosome I

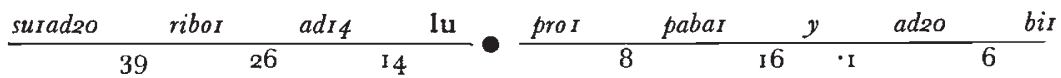

Chromosome II

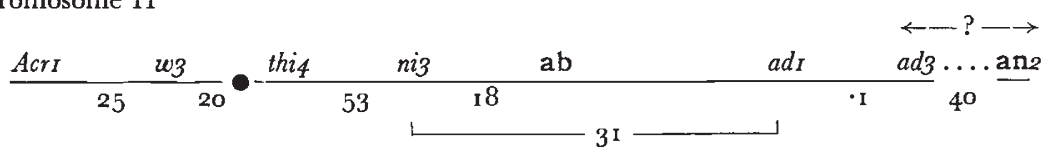

Chromosome III

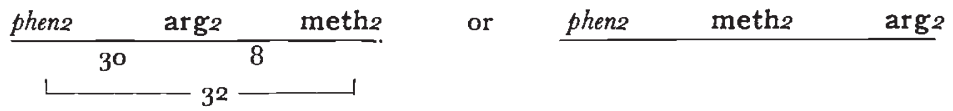

Chromosome IV

\begin{tabular}{llllll} 
methI & & sucI & & pyro4 & orn4 \\
\hline 2 I & & 40 & & 4
\end{tabular}

Chromosome V

lys5

Chromosome VII

nic8
Chromosome VI

$\underline{s 3}$

Chromosome VIII

$\frac{\cos 8}{9}$

Figures represent recombination fractions. (\%)

In bold type, mutants the location of which is the object of the present paper.

Symbols of mutant alleles:

Chromosome I.

suIad2o $=$ suppressor of $a d_{20} . \quad$ ribor $=$ riboflavine requirement.

$a d_{14}=$ adenine requirement. $l u=$ leucine requirement.

proI $=$ proline requirement. pabaI $=p$-amino-benzoica cid requirement. $y=$ yellow

coloured conidia. $\quad a d 2 o=$ partial adenine requirement. $\quad b i_{I}=$ biotin requirement.

\section{Chromosome II.}

$A c r I=$ resistance to acriflavine (incompletely dominant).

$w_{3}=$ white coloured conidia. thi $=$ thiazole requirement.

$n i_{3}=$ nitrite requirement. $a b=$ amino-butyric acid requirement.

$a d_{I}=$ adenine requirement. $a d_{3}=$ adenine requirement.

$a_{n}=$ an eurin requirement.

Chromosome III.

phen $2=$ phenylalanine requirement. $\quad$ arg2 $=$ arginine requirement.

meth $2=$ methionine requirement.

Chromosome IV.

meth $I=$ methionine requirement. suct $=$ inability to grow on sucrose as sole source of carbon. pyro $4=$ pyridoxin requirement.

Chromosome V. lys5 = lysine requirement.

Chromosome VI.

$s_{3}=$ sulphite requirement.

Chromosome VII.

nic $8=$ nicotinic acid requirement.

Chromosome VIII.

$c o=$ compact colony. orn $8=$ ornithine requirement. 
located by using both tester strains, is tentatively assumed to be in the remaining linkage group (VIII).

Tester Strain "A" has the following genotype :-

Chromosome I. Chromosome II. Chromosome III.

su $y$ ad2o

AcrI

phen2

Chromosome IV.

Chromosome V.

Chromosome VII.

pyro 4

lys5

nic8

Tester Strain " B" :-

Chromosome I.

Chromosome II.

Chromosome VI.

su $y$ adro

AcrI

Symbols and linkages are given in the table.

\section{METHODS}

For general techniques and symbols in the genetics of Aspergillus nidulans see Pontecorvo et al. (1953); Pontecorvo and Käfer (1958) and Käfer (1958).

For use in the technique described in this paper the most suitable genotype of a strain with an unlocated mutant, $x$, is $w$ ad2o $x$. However, only a few strains in which an unlocated mutant is present have this genotype. The essential requirement is that ad2o should be present with $x$; the presence of $w$ is desirable but not essential.

Dr E. Käfer (1958) suggested an easy way of introducing adoo into the strain with mutant $x$, provided the latter carries $y^{+}$. The essentials of the technique are as follows. Adiploid between the strain with mutant $x$ and the tester strain, e.g. A. is synthesised (Roper, 1952). This diploid will have the genotype :-

$$
\frac{\text { su y ad2o }}{++t+} \frac{\text { AcrI }}{+} \frac{\text { phen2 }}{+} \frac{\text { pyro4 }}{+} \frac{\text { lys5 }}{+} \frac{\text { nic8 }}{+}\left(\frac{t}{x}\right)
$$

The phenotype will be green (because $y / y^{+}$), partially resistant to acriflavine (because $\left.A_{c r I} /+\right)$, and prototroph because heterozygous for all the nutritional markers. It will be also wild-type in respect of $x$ if $x$ is recessive.

As a first step in the analysis a yellow diploid segregant is selected by inspection from this green prototrophic diploid, and tested for adenine requirement. Mitotic crossing over anywhere between $y$ and the centromere in a diploid $\frac{y \text { adro }}{++}$ will give homozygous yellow diploid segregants which are also homozygous $a d_{20}$, i.e. $\frac{y \text { adzo }}{y \text { adzo }}$. Thus, the genotype of this segregant will be the same as that of the parent diploid except that it is homozygous $\frac{y \text { adzo }}{y \text { adzo }}$.

The second step in the analysis is the selection of haploids from this yellow adenineless diploid segregant, twenty haploids being usually sufficient. To obtain these haploids we can apply the combined selection for adenine independence and for full acriflavine resistance. The third step is to classify haploids in respect of $x$ and the markers on chromosomes III, IV, V and VII.

If $x$ is, for example, on chromosome III, then only the two parental classes of haploids will arise in respect of $x$ and phen2, i.e. $x$ phen $2^{+}$and $x^{+}$phen2. If $x$ is on chromosome pairs IV, V or VII, similarly only the parental classes will arise in respect of $x$ and the marker on one of these chromosome pairs. If $x$ is on chromosome pairs I or II, only $x^{+}$will appear among the selected haploids. If $x$ recombines 
with all markers on chromosome pairs I, II, III, IV, V and VII carried by Tester Strain A, then the use of Tester Strain B will determine if $x$ is on chromosome pair VI. If not, we tentatively conclude that it is on chromosome pair VIII.

As mentioned above, if $x$ is on chromosome pairs I or II, it fails to appear among the selected haploids. We must then find on which of these two pairs it is located. Here the additional presence of $w$ in the strain carrying $x$ is particularly useful. In this case the yellow segregant first isolated will be $\frac{s u \text { adzo }}{+y \text { adro }} \frac{\text { Acr } t}{+w}$, and $x$ will segregate with either $+y$ adro or $+w$. Combined selection of su $y$ adzo $+w$ haploids will show which of the two pairs is involved. If all the haploids so selected are $x$, then $x$ is on chromosome pair II. If all of them are $x^{+}$, then $x$ is on chromosome pair I.

An unlocated mutant phenotypically indistinguishable from one of the markers in the tester strain, but not allelic to it, could be located only if it were on certain chromosome pairs. For instance, if $x$ stood for a mutant determining phenylalanine requirement, not allelic to phen 2 but located on the same chromosome, all the haploids recovered from the homozygous $\frac{y \text { ad } 2 o}{y \text { ad2o }}$ diploid segregant would require phenylalanine due to their being either $x$ or phen2. If $x$ were on the chromosome pair marked in repulsion by pyro4, then all the pyro $4^{+}$would require phenylalanine by virtue of carrying either $x$ or both $x$ and phen2, i.e. pyro $4^{+} x$ phen $2^{+}$or pyro $4^{+} x$ phen2. If $x$ were on the chromosome pair carrying su $y$ adzo or on the chromosome pair carrying AcrI, using the combined acriflavine resistance and adenine independence selection, only $x^{+}$would be recovered among the haploids, but half of those would still require phenylalanine by virtue of phen2. By selecting for white and adenine independence, however, if $x$ were on chromosome pair II then all the haploids would require phenylalanine since $w$ is in coupling with $x$.

This, then, leaves only chromosome pairs I, VI and VIII as alternatives, and chromosome pair VI can be tested by using Tester Strain B. If not located by Tester Strain B only two possibilities remain, $x$ is on chromosome pairs I or VIII.

In order to test the validity of the technique before applying it to unlocated mutants, it was first used with a marker orn4, the location of which on chromosome IV was already known from analysis through sexual reproduction (Käfer, 1958).

\section{EXPERIMENTAL}

\section{Pilot Test with orn4}

The orn 4 mutant determines requirement for ornithine. A balanced heterokaryon was formed between biI ; orn 4 and Tester Strain A. From this, the heterozygous diploid was isolated in the usual way (Roper, 1952) and purified by single conidium micromanipulation. A suspension of conidia from the diploid was then plated onto complete medium so as to give well-separated colonies, and from one of these colonies a homozygous yellow segregant was selected visually. This was then purified by micromanipulation of a single conidium. The strain was tested for ploidy and adenine requirement. A dilute suspension of conidia of the diploid segregant $\frac{y \text { ad2o }}{y \text { ad2o }}$ was next point-inoculated (about five conidia per inoculation, at twenty-six points on a Petri dish) onto minimal medium plus all the growth factors required by each of the parental strains except adenine and biotin (the segregant 
now being homozygous biotin-independent). After 24 hours incubation small spidery colonies had developed with the characteristic sparse growth typical of adzo on adenineless medium. A layer of the same medium plus acriflavine was then poured on top. Another 4 . days incubation was necessary before vigorously growing, wellsporulating segregants had escaped from the mass of partially resistant. hyphæ and had grown through the top layer of medium.

Thirty-four haploids were isolated by picking only one segregant from any one colony to minimise the incidence of repeated isolation of products of the same event. The haploids when fully tested fell into the following classes:

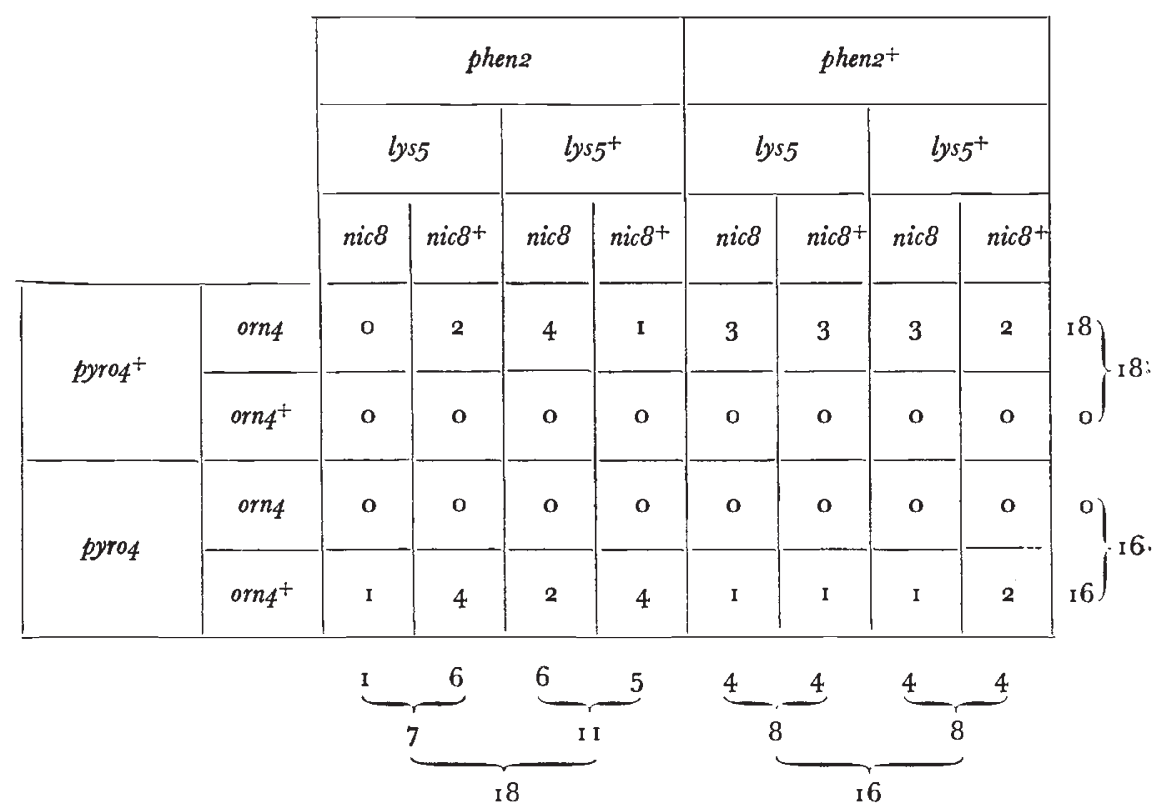

From the results it can be seen that orn 4 shows recombination with all the markers, except pyrou, and is therefore located on the chromosome pair marked by pyro4. The two recombinant classes, pyro4 orn 4 and pyro $4^{+}$orn $4^{+}$, are absent. These are the classes expected to be missing if orn 4 and pyro 4 are linked, since they are in repulsion and haploidisation involves no crossing over. All the markers of the other chromosome pairs segregate independently of one another.

\section{LOCATION OF NEW MUTANTS}

(i) (sucl)

Mitotic Analysis. The unlocated mutant (sucr) determines the inability to grow on sucrose as a sole source of carbon (Roberts, unpublished). A strain biI ; w3; sucI was combined with Tester 
Tester Strain A. Selection on the homozygous $\frac{y \text { ad2o }}{y \text { ad2o }}$ recombinant was again for acriflavine resistance and adenine independence. Thirtyseven haploids were isolated and fully tested.

The haploids fell into the following classes in respect of the combinations of the unlocated mutant with each of the markers identifying the different chromosome pairs :-

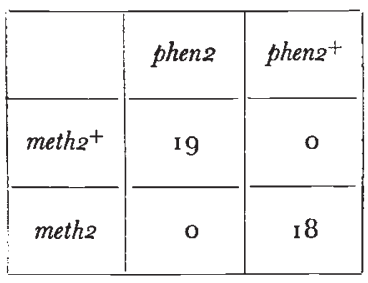

\begin{tabular}{|c|c|c|}
\hline & lys5 & lys5 \\
\hline meth2 $^{+}$ & 8 & 10 \\
\hline meth2 & $\mathrm{I} \mathrm{I}$ & 8 \\
\hline
\end{tabular}

\begin{tabular}{|c|c|c|}
\hline & $n i c 8$ & $n i c 8^{+}$ \\
\hline meth $^{+}$ & 0 & 18 \\
\hline meth2 & 16 & 3 \\
\hline
\end{tabular}

\begin{tabular}{|c|c|c|}
\hline & pyro4 & pyro4 $^{+}$ \\
\hline meth2 $^{+}$ & 9 & 9 \\
\hline meth2 & 9 & 10 \\
\hline
\end{tabular}

The results indicate that meth 2 and phen 2 are on the same chromosome pair.

Meiotic Analysis. From a cross $b i_{I}$; meth $2 \times$ proI pabaI $y$; phen 2 the following relevant results were obtained :-

\begin{tabular}{|c|c|c|}
\hline & phen2 & phen2 ${ }^{+}$ \\
\hline meth2 $2^{+}$ & 37 & 17 \\
\hline meth2 & 13 & 31 \\
\hline
\end{tabular}

Recombination frequency $32 \cdot 5$ per cent.

(iii) (orn8)

Mitotic Analysis. The unlocated mutant (orn8) determines requirement for ornithine. biI ; orn 8 was combined with Tester Strain A and selection on the homozygous $\frac{y \text { ad2o }}{y \text { ad2o }}$ recombinant was again for adenine independence and acriflavine resistance. Twenty-three haploids were isolated and fully tested. The haploids fell into the following classes in respect of the combinations of the unlocated 
mutant with each of the markers identifying the different chromosome pairs :-

\begin{tabular}{|c|c|c|}
\hline & phen2 & phen ${ }^{+}$ \\
\hline orn8 & 5 & 4 \\
\hline orn8 & 7 & 7 \\
\hline
\end{tabular}

\begin{tabular}{|c|c|c|}
\hline & lys5 & lys5 $^{+}$ \\
\hline orn8 $^{+}$ & 4 & 5 \\
\hline orn8 & 6 & 8 \\
\hline
\end{tabular}

\begin{tabular}{|c|c|c|}
\hline & $n i c 8$ & $n i c 8^{+}$ \\
\hline orn $^{+}$ & 6 & 3 \\
\hline orn8 & 5 & 9 \\
\hline
\end{tabular}

\begin{tabular}{|c|c|c|}
\hline & pyro 4 & pyro $4^{+}$ \\
\hline orn $^{+}$ & 3 & 6 \\
\hline orn8 & 5 & 9 \\
\hline
\end{tabular}

The results indicate that orn 8 is not located on any of the chromosome pairs marked by Tester Strain A. Another diploid was therefore synthesised, combining biI ; orn 8 with Tester Strain B and a homozygous $\frac{y \text { ad } 20}{y \text { ad } 20}$ recombinant isolated. The combined selection for adenine independence and acriflavine resistance was applied to this recombinant. Sixteen haploids were isolated, tested and classified as follows :-

\begin{tabular}{|c|c|c|}
\hline & $s_{3}$ & $s 3^{+}$ \\
\hline$-o m 8^{+}$ & 6 & 4 \\
\hline orn8 & 2 & 4 \\
\hline
\end{tabular}

Clearly orn 8 is not located on any of the marked chromosome pairs and by elimination we may assume that it is on chromosome pair VIII.

Meiotic Analysis. From a cross biI ; orn $8 \times p a b a y$; co (the marker co marks chromosome VIII), the following relevant results were obtained :-

\begin{tabular}{|c|c|c|}
\hline & $c o$ & $c 0^{+}$ \\
\hline$o r n 8^{+}$ & 50 & 0 \\
\hline orn8 & 0 & 62 \\
\hline
\end{tabular}

Thus it is confirmed that orn 8 is on chromosome VIII and very closely linked to $c 0$. 


\section{(iv) (an2)}

Mitotic Analysis. The unlocated mutant (an2) determines requirement for aneurin. A strain biI ; AcrI w3; anz was combined with Tester Strain A. Selection on the $\frac{y \text { addoo }}{y \text { ad2o }}$ recombinant was for white and adenine independence. (It will be noted that in this case the original diploid was homozygous AcrI/AcrI). Twenty-one haploids were isolated, tested and all found to require aneurin.

Since all the haploids required aneurin and were white, and white was in the parent strain requiring aneurin, it follows that they are both located on the same chromosome pair.

Meiotic Analysis. From cross biI; AcrI w3; an $\times y$; adI the following relevant results were obtained :-

\begin{tabular}{|c|c|c|}
\hline & $a d I$ & $a d I^{+}$ \\
\hline$a n 2^{+}$ & 28 & 19 \\
\hline$a n 2$ & 19 & 30 \\
\hline
\end{tabular}

Recombination frequency $39^{\circ} 6$ per cent.

\section{(v) (arg2)}

Mitotic Analysis. The unlocated mutant (arg2) determines a requirement for arginine. A strain biI ; arg2 was combined with Tester Strain A. Selection on the homozygous $\frac{y \text { adro }}{y \text { ad2o }}$ recombinant was for adenine independence and acriflavine resistance. Twenty-eight haploids were isolated and fully tested.

The haploids fell into the following classes in respect of the combinations of the unlocated mutant with each of the markers identifying the different chromosome pairs :-

\begin{tabular}{|c|c|c|}
\hline & phen2 & phen2 \\
\hline arg2 $^{+}$ & $\mathrm{II}$ & 0 \\
\hline arg2 & 0 & 17 \\
\hline
\end{tabular}

\begin{tabular}{|c|c|c|}
\hline & lys5 & lys5 \\
\hline arg2 & 5 & 6 \\
\hline arg2 & 7 & 10 \\
\hline
\end{tabular}

\begin{tabular}{|c|c|c|}
\hline & nic8 & nic8 \\
\hline arg2 $^{+}$ & 3 & 8 \\
\hline arg2 & 8 & 9 \\
\hline
\end{tabular}

\begin{tabular}{|c|c|c|}
\hline & pyro4 & pyro4 $^{+}$ \\
\hline arg2 $^{+}$ & 7 & 4 \\
\hline arg2 & 9 & 8 \\
\hline
\end{tabular}


These results indicate that arg2 and phen 2 are on the same chromosome pair.

Meiotic Analysis. From a cross biI; arg $2 \times r i b o 1$ adr $4 y$; phen 2 the following relevant results were obtained :-

\begin{tabular}{|c|c|c|}
\hline & phen2 & phen2 \\
\hline arg2 $^{+}$ & 46 & 27 \\
\hline arg2 & ${ }^{6} 6$ & 40 \\
\hline
\end{tabular}

Recombination frequency 30 per cent.

(vi) (lu)

Mitotic Analysis. The unlocated mutant (lu) determines requirement for leucine. A strain $b i_{I}$; $l u$ was combined with Tester Strain A. Selection on the homozygous $\frac{y \text { ad2o }}{y \text { ad2o }}$ recombinant was for adenine independence and acriflavine resistance. Twenty-one haploids were isolated, tested and found to be all leucine independent. The mutant $l u$ was therefore located on either chromosome pair I or on chromosome pair II. As biI; $l u$ did not carry w3, the selection for white and adenine independence could not be made. This selection would have made it possible to confirm on which of these two chromosome pairs lu was located.

Meiotic Analysis. Two crosses were set up with the lu mutant. One cross was to a strain with chromosome I extensively marked and the other to a strain with chromosome II extensively marked. Meiotic analysis from the cross of biI; lu $\times$ ribor adI 4 proI pabaI $y$ gave the following relevant results :-

\begin{tabular}{|c|c|c|}
\hline & $a d_{\mathrm{I} 4}$ & $a d_{\mathrm{I} 4^{+}}$ \\
\hline$l u^{+}$ & 63 & $\mathrm{I} 4$ \\
\hline$l u$ & 7 & 63 \\
\hline
\end{tabular}

Recombination frequency I 4.3 per cent.

\begin{tabular}{|c|c|c|}
\hline & ribor & ribot ${ }^{+}$ \\
\cline { 2 - 3 }$l^{+}$ & 49 & $\mathrm{I} 4$ \\
\hline$l u$ & 24 & 43 \\
\hline
\end{tabular}

Recombination frequency 30 per cent.

The cross to the strain with marked chromosome II was therefore not analysed.

Mitotic Analysis. The unlocated mutant (ab) determines requirement for alpha-amino-n-butyric acid. A strain $b i r ; a b$ was combined with Tester Strain A. Selection on the homozygous $\frac{y \text { ad2o }}{y \text { ad2o }}$ recombinant 
was for adenine independence and acriflavine resistance. Forty-two haploids were isolated and fully tested. All were amino-butyric acid independent. As in the case of the $l u$ mutant, selection for white and adenine independence could not be made.

Meiotic Analysis. Two crosses were set up, one to a strain with chromosome I extensively marked and the other to a strain with chromosome II extensively marked. Meiotic analysis from the cross bir; $a b \times$ ribor bil ; Acrr w3 thi4 $n i_{3}$ ad 3 gave the following relevant results :-

\begin{tabular}{|c|c|c|}
\hline & $a d 3$ & $a d 3^{+}$ \\
\hline$a b^{+}$ & $4^{6}$ & 16 \\
\hline$a b$ & 25 & 45 \\
\hline
\end{tabular}

Recombination frequency 3 i per cent.

\begin{tabular}{|c|c|c|}
\hline & $n i 3$ & $n i 3^{+}$ \\
\hline$a b^{+}$ & 52 & 10 \\
\hline$a b$ & 13 & 57 \\
\hline
\end{tabular}

Recombination frequency 18 per cent.

\section{CONCLUSIONS}

Of the eight mutants located by means of the haploidisation technique described in this paper, only one was meiotically closely linked to the relevant marker in the tester strains.

Four of the other seven were loosely linked meiotically with the marker of the tester strain relevant in each case. The remaining three showed no meiotic linkage with the relevant marker, but did show it with other markers meiotically linked to the relevant marker. Thus the efficiency of the technique is such that an unlocated mutant can be assigned to the linkage group to which it belongs even though the marker identifying that linkage group and the unlocated mutant are more than fifty meiotic map units apart.

Two further improvements of the technique are possible :-

(I) The use of a single tester strain with at least seven of the eight chromosomes marked instead of two tester strains.

(2) The use for combined selection of two markers, one on each arm of the same chromosome instead of one on each of two non-homologous chromosomes. A new marker $x$ on the homologue of the "selector" chromosome would show its location on that chromosome because only $x^{+}$would appear among the selected haploids. With the technique used so far, on the other hand, the recovery of $x^{+}$leaves two alternative locations, i.e. on either of the two chromosome pairs used for selection.

An example of (2) is the following :-

For the isolation of new mutants, a strain $y^{+}$adro is used. The diploid combination of a new mutant and a tester strain will be

$$
\frac{s u}{+} 0 \frac{y \text { ad } 20}{+a d 2 o} \frac{+}{x}
$$


Visual selection of yellow segregants on adenineless medium will yield haploids of constitution su y adro.

With the use of this modified technique (combined selection for two markers, one on each arm of the same chromosome) we might expect that among the selected segregants the proportion of diploids should be somewhat higher than in the case of combined selection for two markers one in each of two non-homologous chromosomes. This is because of the rare occurrence of non-disjunction in individual chromosome pairs (see Pontecorvo and Käfer, I958, p. 89), a trouble not likely to reduce substantially the efficiency of the technique.

\section{SUMMARY}

I. A technique based on mitotic segregation has been devised for quickly and easily assigning new mutants to their linkage groups in Aspergillus nidulans.

2. The technique is based on the fact that strains of $A$. nidulans with diploid nuclei produce haploids vegetatively as a rare but regular event. As the process of mitotic haploidisation is known not to be associated with mitotic crossing over, the individual members of each chromosome pair segregate as whole units. Thus, among such haploids, markers on different chromosome pairs show free recombination, while markers on any one chromosome pair do not recombine.

3. Heterozygous diploids are prepared between a suitable strain carrying the new mutant and two tester strains, marking between them seven of the eight chromosome pairs. Haploid segregants are selected automatically by a number of devices which distinguish them from those segregants, still diploid, which arise by mitotic crossing over.

4. Seven new markers on five chromosome pairs have been located by this technique. Subsequent meiotic analysis has confirmed each of these locations.

Acknowledgments. - The author would like to express his thanks to Professor G. Pontecorvo, F.R.s., for his criticism and advice both during the work and, especially, in the preparation of this paper.

This work is part of a programme supported by the Nuffield Foundation.

\section{REFERENCES}

KAfER, E. 1958. An 8-chromosome map of Aspergillus nidulans. Adv. Genet., 9, 105-145.

PONTECORVO, G., AND KÄFER, E. 1956. Mapping the chromosomes by means of mitotic recombination. Proc. Roy. phys. Soc. (Edin.), 25, i6-20.

PONTECORVO, G., AND KÄFER, E. I958. Genetic analysis based on mitotic recombination. Adv. Genet., 9, 71-104.

PONTECORVO, G., ROPER, J. A., HEMMONS, L. M., MACDONALD, K. D., AND BUfTON, A. w. J. 1953. The genetics of Aspergillus nidulans. Adv. Genet., 5, 141-238.

PONTECORVO, G., TARR-GLOOR, E., AND FORBES, E. 1954. Analysis of mitotic recombination in Aspergillus nidulans. F. Genet., 52, 226-237. 
PRITCHARD, R. H. I955. The linear arrangement of a series of alleles of Aspergillus nidulans. Heredity, 9, 343-371.

ROPER, J. A. 1952. Production of heterozygous diploids in filamentous fungi. Experientia, 8, I4-I5.

ROPER, J. A., AND KÄFER, E. 1957. Acriflavine resistant mutants in Aspergillus nidulans. 7. Gen. Microbiol., $16,660-667$. 